\title{
ESTEROIDES PRODUZIDOS POR Penicillium herquei, UM FUNGO ENDOFÍTICO ISOLADO DOS FRUTOS DE Melia azedarach (MELIACEAE)
}

\author{
Andrey Moacir do Rosario Marinho* \\ Departamento de Ciências Naturais, Universidade do Estado do Pará, 6113-010 Belém - PA, Brasil \\ Patrícia Santana Barbosa Marinho e Edson Rodrigues Filho \\ Departamento de Química, Universidade Federal de São Carlos, 13565-905 São Carlos - SP, Brasil
}

Recebido em 30/4/08; aceito em 18/3/09; publicado na web em 4/8/09

\begin{abstract}
STEROIDS PRODUCED BY Penicillium herquei, AN ENDOPHYTIC FUNGUS ISOLATED FROM THE FRUITS OF Melia azedarach (MELIACEAE). Six compounds comprising the groups of steroids, the ergosterol, the ergosterol peroxide, the cerevisterol, the neociclocitrinols, the ergosta-4,6,8(14),22-tetraen-3-one, the 25-hydroxy-ergosta-4,6,8(14),22-tetraen-3-one, were isolated from Penicillium herquei fungus obtained from Melia azedarach. The structures were identified by spectral methods of RMN 1D and 2D and MS.
\end{abstract}

Keywords: steroids, endophytic fungus, Penicillium.

\section{INTRODUÇÃO}

Micro-organismos endofíticos estão presentes nos tecidos internos das plantas onde esta associação pode ocorrer em nível celular. É estimado que cerca de $80 \%$ das plantas são hospedeiras de fungos. ${ }^{1-3}$ A natureza dessas interações tem despertado o interesse de químicos, bioquímicos e biólogos na tentativa de entender essas associações, que é um fascinante e amplo campo de pesquisa. A colonização das plantas por endofíticos pode ser mediada pelo seu metabolismo secundário. ${ }^{4}$ Em geral esses compostos apresentam atividades antimicrobianas e inseticidas, e sua produção pode ser uma forma de proteção da planta hospedeira contra invasões. ${ }^{1,4}$

Nosso grupo tem estudado a química de micro-organismos associados a plantas da família Meliaceae. Uma grande coleção de fungos tem sido isolada das folhas, frutos, raiz e caule de Melia azedarach. ${ }^{5}$ Algumas espécies de Penicillium isoladas dessa planta têm produzido metabólitos secundários interessantes pela sua química e atividade biológica. ${ }^{6}$ Este artigo descreve o estudo químico de $P$. herquei, cultivado em arroz, o isolamento e identificação de esteroides.

\section{RESULTADOS}

As substâncias 1, 2 e $\mathbf{3}$ foram obtidas da fase diclorometânica do extrato metanólico da biomassa produzida por $P$. herquei cultivado em arroz (Uncle Ben's). Essas substâncias são sólidas cristalinas e seus dados de RMN e EM são perfeitamente coincidentes com os descritos na literatura para o ergosterol (1), peróxido de ergosterol (2) e cerevisterol (3). . $^{7-9}$

$\mathrm{O}$ espectro de $\mathrm{RMN}{ }^{1} \mathrm{H}$ de 4 não apresentou características de um composto com esqueleto esteroidal comum. Seu espectro de EM (APCI) apresentou um $\mathrm{m} / \mathrm{z} 401[\mathrm{M}+\mathrm{H}]^{+}$. Foram observados ainda

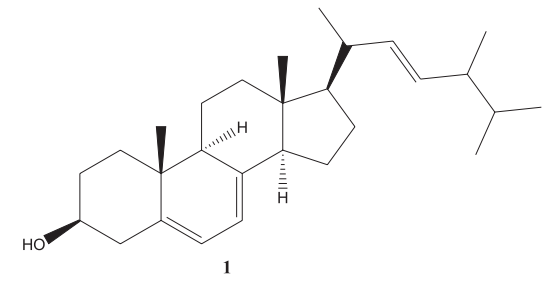

*e-mail: andreymoacir@yahoo.com.br
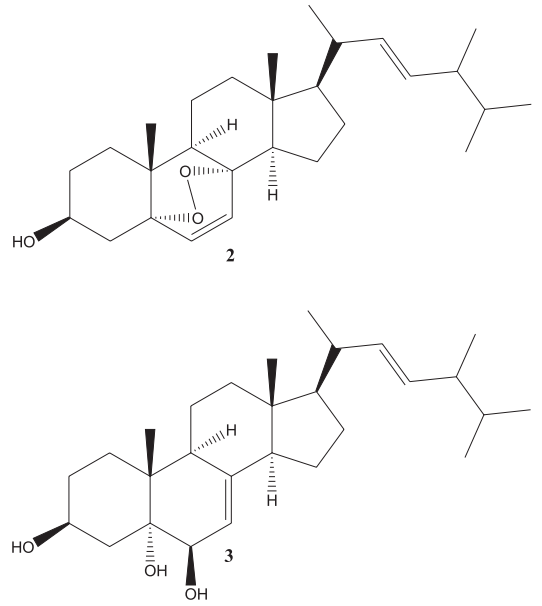

os $m / z$ 383, 365 e 347 indicando a perda de três moléculas de água, sugerindo uma molécula tri-hidroxilada. $\mathrm{O}$ espectro de $\mathrm{RMN}{ }^{1} \mathrm{H}$ apresentou sinais para três hidrogênios carbinólicos H-3 $(\delta 3,24), \mathrm{H}-23$ ( $\delta$ $4,18 / 4,04)$ e H-24 $(\delta 3,60 / 3,47)$; estas informações corroboram com as perdas de água no espectro de massas e observa-se ainda no espectro de $\mathrm{RMN}{ }^{13} \mathrm{C}$ um sinal típico de carbonila em $\delta$ 207,8 (C-6). Foram evidentes alguns sinais duplicados com relação 1:1 de intensidade. Estes dados sugeriram a presença de uma mistura epimérica. Após análise dos dados de RMN e EM concluímos que 4 é a mistura das formas 23,24-eritro e 23,24-treo do esteroide neociclocitrol isolado anteriormente por nós de Penicillium janthinellum. ${ }^{10}$ Não foi possível separar a mistura por nenhum método de HPLC. Recentemente foi isolada uma série de esteroides neociclocitrinois de $P$. citrinum e o estudo biossintético realizado neste trabalho corroborara com a nossa proposta de rota bioquímica. ${ }^{10,11}$

Os espectros de $\mathrm{RMN}{ }^{1} \mathrm{H}$ e ${ }^{13} \mathrm{C}$ da substância 5 apresentaram características de um composto esteroidal. No espectro de $\mathrm{RMN}{ }^{1} \mathrm{H}$ observamos o sinal de grupo metila $\mathrm{CH}_{3}-18(\delta 0,95, s, 3 \mathrm{H})$ ligada a um carbono quaternário. Seguindo as correlações para esta metila foi possível determinar os sinais dos carbonos C-12 ( $\delta 35,7), C-13$ $(\delta 44,1), \mathrm{C}-14(\delta 156,0)$ e C-17 ( $\delta 55,8)$. O sinal $\delta 0,98$ foi atribuído à metila $\mathrm{CH}_{3}-19$ e tem correlações de $\mathrm{HMBC}$ com C-2 ( $\left.\delta 34,2\right)$, 


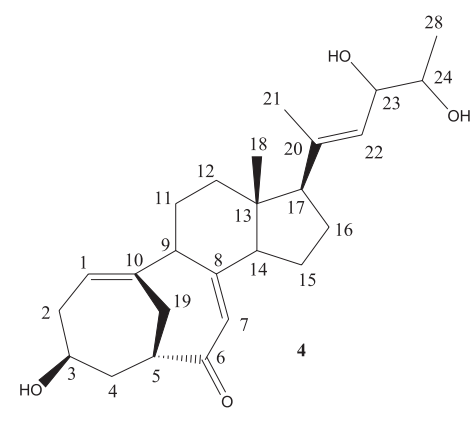

C-10 $(\delta 36,8)$, C-9 $(\delta$ 44,5) e C-5 $(\delta$ 164,2). Os hidrogênios H-6 $(\delta 6,01)$ e H-7 $(\delta 6,60)$ apresentaram constante de acoplamento $J$ $=9,6 \mathrm{~Hz}$ e devem estar cis relacionados. As correlações de HMBC e COSY para $\mathrm{CH}_{3}-18, \mathrm{CH}_{3}-19, \mathrm{H}-6$ e $\mathrm{H}-7$ permitiram definir um sistema conjugado 4,6,8(14) nos anéis A, B e C. O sinal em $\delta 5,72$ apresentou correlação no HMBC com os carbonos C-5, C-6, C-10 e foi atribuído a H-4. Esta atribuição pode ser confirmada através da correlação com a carbonila $\alpha, \beta$-insaturada localizada em C-3 ( $\delta$ 199,3). Através das análises dos dados de RMN, EM e comparações com os dados descritos na literatura concluímos que a substância 5 é o ergosta-4,6,8(14),22-tetraen-3-ona.

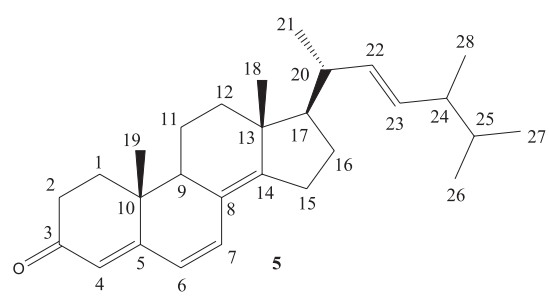

A substância 6 apresentou o mesmo padrão de correlações no HMBC, HSQC e COSY para os anéis A, B, C e D da substância 5. Porém ao invés de quatro sinais dubletos de metila como em $\mathbf{5}$ foram observados dois sinais dubletos e quatro sinais singletos na região de metilas no espectro de $\mathrm{RMN}{ }^{1} \mathrm{H}$ de 6 . No espectro de $\mathrm{RMN}{ }^{13} \mathrm{C}$ de $\mathbf{6}$ observou-se ainda um sinal referente a um carbono carbinólico em $\delta$ 72,5. Como os anéis A, B, C e D são idênticos aos de 5, o carbono carbinólico foi localizado na cadeia lateral. Já que no espectro de $\mathrm{RMN}{ }^{1} \mathrm{H}$ são observados quatro sinais referentes a singletos de metila, o grupo hidroxila foi posicionado em C-25, que é o único local na cadeia lateral que contempla o padrão espectral observado. Essa proposta foi confirmada através da correlação da metila $\mathrm{CH}_{3}-28$ no HMBC com C-23 e $\delta 72,5$ (C-25). As metilas $\mathrm{CH}_{3}-26$ e $\mathrm{CH}_{3}-27$ apresentaram correlação com C-24 $(\delta 49,0)$ e com C-25 confirmando a estrutura de $\mathbf{6}$. Assim, 6 foi identificado como 25-hidroxi-esgosta4,6,8(14),22-tetraen-3-ona.

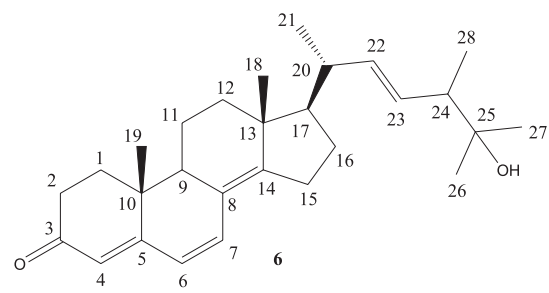

As substâncias 5 e $\mathbf{6}$ foram isoladas anteriormente de uma espécie de Ascomiceto encontrado em uma esponja. ${ }^{12,13}$ Esta é a primeira ocorrência desses esteroides em espécies de Penicillium. Os dados de $\mathrm{RMN}{ }^{1} \mathrm{H}$ e ${ }^{13} \mathrm{C}$ de 5 e $\mathbf{6}$ estão dispostos da Tabela 1.
Tabela 1. Dados de $\mathrm{RMN}{ }^{1} \mathrm{H}^{*}(400 \mathrm{MHz})$ e ${ }^{13} \mathrm{C}(100 \mathrm{MHz})$ de 5 e 6 em $\mathrm{CDCl}_{3}$

\begin{tabular}{|c|c|c|c|c|}
\hline \multirow{2}{*}{ Posição } & \multicolumn{2}{|c|}{5} & \multicolumn{2}{|c|}{6} \\
\hline & $\delta{ }^{1} \mathrm{H}$ & $\delta^{13} \mathrm{C}$ & $\delta^{1} \mathrm{H}$ & $\delta^{13} \mathrm{C}$ \\
\hline 1 & & 34,1 & & 34,1 \\
\hline 2 & & 34,2 & & 34,2 \\
\hline 3 & & 199,3 & & 199,5 \\
\hline 4 & $5,72(s)$ & 123,1 & $5,74(s)$ & 123,5 \\
\hline 5 & & 164,2 & & 164,3 \\
\hline 6 & $6,01(d, 9,4)$ & 124,5 & $6,04(d, 9,7)$ & 124,5 \\
\hline 7 & $6,60(d, 9,4)$ & 133,9 & $6,60(d, 9,7)$ & 134,0 \\
\hline 8 & & 124,5 & & 124,4 \\
\hline 9 & & 44,5 & & 44,5 \\
\hline 10 & & 36,8 & & 36,8 \\
\hline 11 & & 19,0 & & 19,0 \\
\hline 12 & & 35,7 & & 35,6 \\
\hline 13 & & 44,1 & & 44,1 \\
\hline 14 & & 156,0 & & 156,0 \\
\hline 15 & & 25,4 & & 25,4 \\
\hline 16 & & 27,7 & & 27,7 \\
\hline 17 & & 55,8 & & 56,1 \\
\hline 18 & $0,95(s)$ & 19,0 & $0,97(s)$ & 19,0 \\
\hline 19 & $0,98(s)$ & 16,7 & $1,00(s)$ & 16,2 \\
\hline 20 & & 39,2 & & 39,6 \\
\hline 21 & $1,05(d, 6,7)$ & 21,2 & $1,08(d, 6,7)$ & 21,1 \\
\hline 22 & $5,22(m)$ & 135,0 & $5,37(\mathrm{~m})$ & 138,2 \\
\hline 23 & $5,22(m)$ & 132,6 & $5,37(m)$ & 129,9 \\
\hline 24 & & 42,9 & & 49,0 \\
\hline 25 & & 33,1 & & 72,5 \\
\hline 26 & $0,82(d, 6,8)$ & 20,0 & $1,15(s)$ & 27,0 \\
\hline 27 & $0,84(d, 6,8)$ & 19,7 & $1,18(s)$ & 26,3 \\
\hline 28 & $0,92(d, 6,8)$ & 17,6 & $1,01(d, 6,9)$ & 15,5 \\
\hline
\end{tabular}

${ }^{*}$ Multiplicidades e constantes de acoplamento $(J)$, em Hz, entre parênteses

\section{PARTE EXPERIMENTAL}

\section{Procedimentos gerais}

O espectro de IV foi medido em um espectrofotômetro Bomen MB-102 em pastilha de KBr. Os dados de APCIMS foram adquiridos no modo positivo usando um instrumento Micromass Quattro-LC equipado com uma fonte de íons ESI/APCI do tipo Z-spray. Os experimentos de $\mathrm{RMN}{ }^{1} \mathrm{He} \mathrm{e}^{13} \mathrm{C}$ foram obtidos em um espectrômetro Bruker DRX-400 em MeOD e $\mathrm{CDCl}_{3}$ com TMS como padrão interno.

\section{Micro-organismo}

$P$. herquei foi obtido de uma coleção do Laboratório de Bioquímica Micromolecular de Micro-organismos do Departamento de Química da Universidade Federal de São Carlos. Esta coleção contém isolados de Melia azedarach. ${ }^{5}$ 


\section{Cultura de $P$. herquei em arroz e isolamento dos esteroides}

Quarenta e cinco fracos de Erlenmeyer (500 mL), contendo $90 \mathrm{~g}$ de arroz (Uncle Been's) e $75 \mathrm{~mL}$ de água destilada por frasco, foram autoclavados por $45 \mathrm{~min}$ a $121^{\circ} \mathrm{C}$. Pequenos cubos de meio BDA contendo o micélio de $P$. herquei foram adicionados em 42 frascos de Erlenmeyer sob condição estéril. Três frascos foram utilizados como controle. Após 20 dias de crescimento a $25{ }^{\circ} \mathrm{C}$ foi adicionado metanol (300 mL) em cada frasco de Erlenmeyer, e após 5 h esse metanol foi filtrado por gravidade. O metanol foi evaporado sob pressão reduzida, onde se obteve 10,4 g de extrato. Esse extrato foi fracionado em coluna sob pressão reduzida usando hexano, diclorometano e metanol como eluentes. Da fase diclorometânica, após fracionamento em coluna cromatográfica de sílica gel eluída com hexano, acetona e metanol, em gradiente de polaridade, foram obtidos os esteroides ergosterol (1), peróxido de ergosterol (2), cerevisterol (3), neociclocitrinois (4), ergosta-4,6,8(14),22-tetraen-3-ona (5) e 25-hidroxi- ergosta-4,6,8(14),22-tetraen-3-ona (6).

\section{Dados físicos e espectrais dos compostos}

Ergosta-4,6,8(14),22-tetraen-3-ona (5): sólido amarelo pálido; $\mathrm{RMN}{ }^{1} \mathrm{He} \mathrm{e}^{13} \mathrm{C}\left(\mathrm{CDCl}_{3}, 400 \mathrm{MHz}\right)$ ver Tabela 1; APCIMS (full scan, modo positivo): $m / z$ (rel. int): $393[\mathrm{M}+\mathrm{H}]^{+}$(100), 346 (31), 306 (75), 252 (52).

25-hidroxi-ergosta-4,6,8(14),22-tetraen-3-ona (6): sólido amarelo pálido; $\mathrm{RMN}{ }^{1} \mathrm{H} \mathrm{e} \mathrm{e}^{13} \mathrm{C}\left(\mathrm{CDCl}_{3}, 400 \mathrm{MHz}\right)$ ver Tabela 1; APCIMS (full scan, modo positivo): $\mathrm{m} / \mathrm{z}$ (rel. int): $409[\mathrm{M}+\mathrm{H}]^{+}$(100); APCIMS (íons filhos, $30 \mathrm{eV}$ ): 409 [M+H] ${ }^{+}$(20), 391 (35), 349 (28), 333 (38), 253 (88), 235 (65), 225 (100), 211 (38), 199 (38).

\section{AGRADECIMENTOS}

À Fundação de Amparo à Pesquisa do Estado de São Paulo (FAPESP), Conselho Nacional de Desenvolvimento Científico e Tecnológico (CNPq) e Coordenação de Aperfeiçoamento de Pessoal de Ensino Superior (CAPES) pelo suporte financeiro.

\section{REFERÊNCIAS}

1. Rizzo, I.; Varsavky, M.; Haidukowski, M.; Frade, H.; Toxicon 1997, 35, 753.

2. Stierle, A.; Strobel, G.; Stierle, D.; Science 1993, $260,214$.

3. Zhang, W.; Wendel, J. F.; Clark, L. G.; Mol. Phylogent. Evol. 1997, 8, 205.

4. Huang, E. X.; Huang, T. L.; Wildung, M. R.; Croteau, R., Scott, A. I.; Prot. Expr. Pur. 1998, 13, 90.

5. Santos, R. M. G.; Rodrigues-Fo, E.; Rocha, W. C.; Teixeira, M. F. S.; World J. Microbiol. Biotechnol. 2003, 19, 767.

6. Marinho, A. M. do R.; Rodrigues-Fo, E.; Santos, L. S.; Moitinho, M. L. R..; J. Braz. Chem. Soc. 2005, 16, 280.

7. Breitmaier, E.; Voelter, W.; Carbon-13 NMR Spectroscopy: High Resolution Methods and Applications in Organic Chemistry and Biochemistry, $3^{\text {rd }}$ ed., VCH: Weinhein, 1987.

8. Yue, J.; Chen, S.; Lin, Z.; Sun, H.; Phytochemistry 2001, 56, 801.

9. Kawagishi, H.; Katsumi, R.; Sazawa Takashi, M.; Hagiwara, T.; Nakamura, T.; Phytochemistry 1988, 27, 2777.

10. Marinho, A. M. R.; Rodrigues-Fo, E.; Ferreira, A. G.; Santos, L. S.; J. Braz. Chem. Soc. 2005, 16, 1342.

11. Du, L.; Zhu, T.; Fang, Y.; Gu, Q.; Zhu, W.; J. Nat. Prod. 2008, 71, 1343.

12. Fujimoto, H.; Nakamura, E.; Okuyama, E.; Ishibashi, M.; Chem. Pharm. Bull. 2004, 52, 1005 .

13. Ciminiello, P.; Fattorusso, E.; Magno, S.; Mangoni, A.; J. Nat. Prod. 1989, 52, 1331. 